Borneo Journal of Sciences and Technology, Volume (1), Issue (1), Pages: 36-40

DOI: https://doi.org/10.35370/bjost.2019.1.1-08

e-ISSN: 2672-7439

(C) 2018, UCTS Publisher.

Submitted: 31 October 2018

Accepted: 10 November 2018

Published: 31 January 2019

\title{
A review about Zig Zag Connections in Transformers
}

\author{
Geno Peter, Samat bin Iderus, Ramli bin Rashidi
}

School of Engineering and Technology, University College of Technology Sarawak, Jalan Universiti, 96000 Sibu, Sarawak, Malaysia

\begin{abstract}
Whenever, we think of three phase transformer connections, we think straightaway either about star connected transformer or delta connected transformers. A great majority of polyphase transformers are either star connected or connected in delta. In either case, the connection is symmetrical or balanced. In this paper we wish to explore about zig zag windings being used as earthing transformers and the effect of short circuit current. Double zig zag windings is also dealt here.
\end{abstract}

Keywords: Transformer, Star, Delta, Polyphase, Zig Zag windings

\section{INTRODUCTION}

Let ' $E$ ' represent the three phase line voltage and ' $I$ ' the three phase line current, then for delta connection, each phase receives a voltage ' $\mathrm{E}$ ' and current ' $\mathrm{I} / 1.73^{\text {' }}$.For the star connection, each phase receives a voltage 'E/1.73' and a current ' $I$ '. In either case, the rating of each phase is ' $\mathrm{EI} / \sqrt{3}$ ' $^{\text {' and hence }}$

$$
\text { Rating of the transformer }=\sqrt{ } 3 \text { EI }
$$

which is the same as the power associated with a line voltage ' $E$ ' and a line current ' $I$ ' in a three phase system. Hence it follows that kVA of such three phase star or delta connected transformers is equal to $\mathrm{kVA}$ delivered to load circuit (except influence of magnetizing current and regulation drop). [1] Hence ratio of $\mathrm{kVA}$ load to $\mathrm{kVA}$ of transformer is unity. The zig zag winding connections is used as earthing transformer. Its applications are for the creation of a missing neutral connection from an ungrounded 3phase system to permit the grounding of that neutral to an earth reference point and also harmonic mitigation, as it can suppress triplet (3rd, 9th, 15th, 21st, etc.) harmonic currents.

\section{WHAT IS ZIG ZAG CONNECTION?}

The fact that in all other three phase transformer systems is that the ratio is less than unity probably account for the preference of star or delta connections, over all other connections. Sometimes, polyphase transformers are arranged with windings in different legs connected in series; one type of such interconnection is shown in fig.1. For convenience, we can assume it as an auto transformer or a transformer with the second winding not shown. The winding shown parallel to each other are on the same leg i.e., for example 'aal' and 'a1n' are on the same leg. In this case, all windings having the same relative polarity are connected.

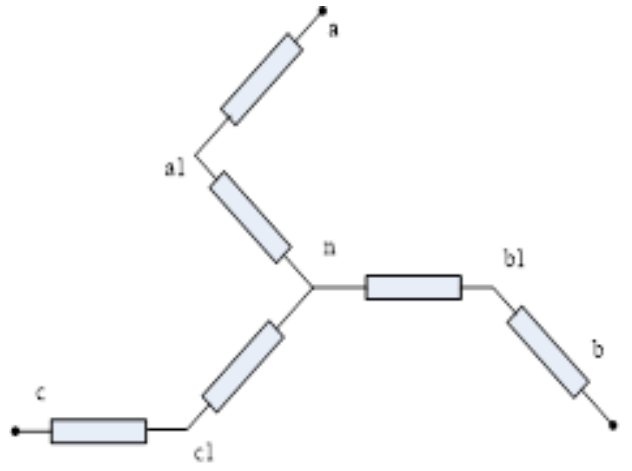

Figure 1. Zig Zag connection

The most important thing is that the circuit is asymmetrical. This connection is called the interconnected star or Zigzag connection. We see that in a Zigzag connection, the net magnetomotive force is $(\sqrt{3} / 2)$ or 0.866 of what it would be if two windings on the same leg were connected in seri 


\section{COMPARISON BETWEEN STAR AND ZIG ZAG CONNECTION}

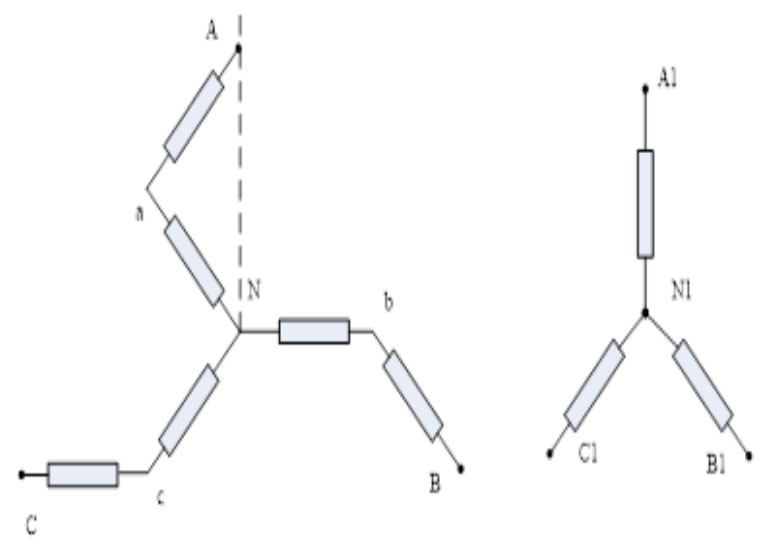

Figure 2. Star/ Zig Zag connection

Comparing to a star connected winding for the sake of analysis, we can write the following on a phase to phase basis.

'A1N1' from star winding = 'AN' from inter star winding,

where , $\mathrm{A} 1 \mathrm{~N} 1$ ' is the voltage directly associated with the winding in star, ' $\mathrm{AN}$ ' in the case of Zig Zag winding is the vectorial sum of two voltages represented by the voltages in windings ' $\mathrm{Aa}$ ' and ' $\mathrm{Na}$ '.

Assume ,'A1N1'has '2W'turns. Let 'Aa' = W turns and ' $\mathrm{aN}$ ' = W turns.

Then 'AN' = sum of voltages in 'Aa' and ' $\mathrm{Na}$ '. i.e.

$\mathrm{W}(0.866)+\mathrm{W}(0.866)$ as voltage proportional to turns $=1.732 \mathrm{~W}$.

Voltage 'A1N1' = '2W' assuming same voltage.

Hence in Zig Zag connection the induced line to neutral voltage for a given core flux is 0.866 times the value for a star connection. In other words, for a given line to neutral voltage, 1.15 times as many winding turns are required when windings are connected in $\mathrm{Zig}$ Zag. [2] The other important point is that there is a phase displacement of $30^{\circ}$ degrees between the line to neutral voltages. Hence for the same number of turns as a star winding, we can say that Zig Zag connections are capable of delivering only $86.6 \%$ of the total transformer rating.

Another important basis for comparison is the question of symmetry with respect to line and neutral. Voltage and current symmetry, both with respect to the three lines and also lines to neutral is obtainable only in the delta and Zig Zag connections. The star connection, although symmetrical as far as lines are connected, introduces third harmonic current and voltage asymmetry between lines and neutral, which may be responsible for causing dangerous over voltages or possibly interference with telephone circuit. One of the big advantages of star winding is the availability of a neutral point. The other advantage is the increased copper cross section for winding compared to delta winding has the advantage of providing the balancing ampere turns for third harmonic and Zero phase sequence flux.

Both these advantages are available with the inter connected star transformer. By means of the Zig Zag winding, an inherently stable neutral can be derived permitting angle phase line to neutral loads free from harmonic residuals. But it must be remembered that only the neutral of Zig Zag winding is stabilized if the other winding in the transformer is a star winding. But practically we see that inter connected star windings are made use of on a regular basis, only for distribution transformers, up to about $1600 \mathrm{kVA}$.

\section{APPLICATION OF ZIG ZAG WINDING}

The principal application of the Zig Zag winding is in the so-called earthing or grounding transformer. The basic purpose of a grounding transformer is to convert an otherwise isolated neutral system into a grounded neutral system. Looking another way, the purpose is to make available a grounded neutral for a system having no neutral.

If the entire system to which the inter connected auto transformer is connected, is symmetrical. The lines to neutral voltages of the Zig Zag transformer are balanced. The only currents flowing in to the transformers are the magnetizing currents, for exciting the core.

For an earth fault, the resulting currents flowing in the windings are indicated in the fig.3. The six coil currents are equal and each is equal to $1 / 3$ of the ground current. As the coil currents in the upper and lower coils in a core leg are not only equal but opposite, their combined magnetic effect is the leakage reactance between the two coils. [3] 


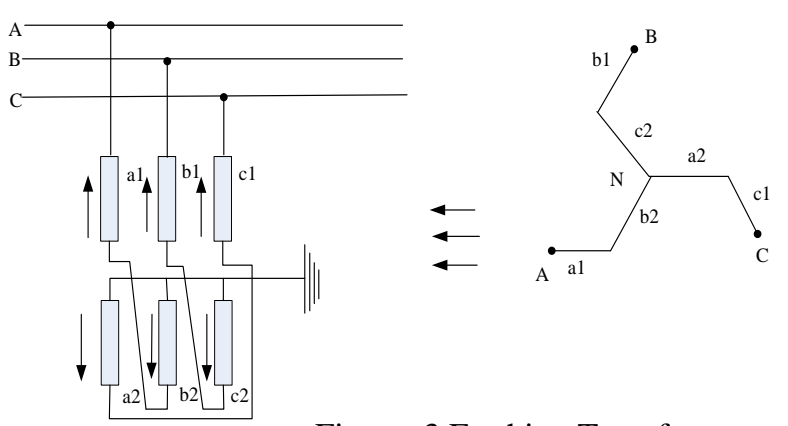

Figure. 3 Earthing Transformer

With a dead ground on one of the lines, a short circuit current flowing in every coil will be

$$
\text { Is }=(1.732 \times \mathrm{I}) \div \% \mathrm{IZ}
$$

Where $\mathrm{I}=$ rated current of coils.

$\% \mathrm{IZ}=$ Impedance between coils calculated for rated current I and expressed in percentage of normal coil voltage (which is equal to $1 / 3$ of line to line voltage).

It will be found that impedance drops of all the three phases are identical, during the fault. The Zig Zag winding transformer consists of six equal windings, each designed for $1 / 3$ of the line voltage and designed to carry the short circuit current.

The total short circuit volt-amperes associated with transformer is

$$
((6 \times E) \div(3 \times I s))=2 \times E \times I s
$$

where $\mathrm{E}$ is the line voltage. This value is $57.5 \%$ of that of far the star delta grounding transformer for the same performance. A star - delta transformer requires two windings to perform the same task as a one winding Zig Zag winding transformer. The advantage of a star delta transformer is that in addition to performing as a grounding transformer in times of fault, it can also supply auxiliary loads, when the delta is designed suitably for the load voltages. Hence, to make better use of the Zig Zag grounding transformer, it is sometimes a practice to provide an auxiliary winding up to a maximum of about $500 \mathrm{kVA}$.

The winding for the neutral current is noninductive (except for leakage reactance) will be evident considering the fact that the ampere turns in each phase is balanced. Thus ampere turns of coil ,'a1' , are balanced by those of ' $\mathrm{a} 2^{\text {', }}$, those in 'b1', by ' $\mathrm{b} 2^{\text {' }}$ ' and those in 'c1', by those of ' ${ }^{2}$ ', without producing a flux through the complete core, but only through the leakage space between each pair of coils.

The ratings of these units will be entirely based on the line voltage and the unbalanced current in the neutral, but has no direct relation to the balanced three phase load current, as this does not flow through them. When the neutral is grounded, the $\mathrm{kVA}$ rating of these units is based on the value of short circuit current they are expected to handle and the duration of such currents. The leakage reactance of the unit and that of the system should then be considered to make sure that the actual short circuit current will not exceed the specified value.

\section{DIFFERENCE BETWEEN CONVENTIONAL AND EARTHING TRANSFORMER}

One of the principal differences between a conventional transformer and a grounding transformer is in the durations of short circuit currents to be handled. For conventional transformers, as per established international standards, it may be 2 to 3 seconds, and for a grounding transformer it may be from 3 to 60 seconds. So, the standard temperature limit of $250^{\circ} \mathrm{C}$ established for standard transformer is not applicable in case of grounding transformers. For short circuits of longer duration, the temperature rise limit should be reduced based on duration and frequency of such faults. [4] under,

An approximate thumb rule for the temp. rises is as

Table 1. The temperature rises during short circuit

\begin{tabular}{|lllll|l|}
\hline $\begin{array}{l}\text { Duration } \\
\text { seconds }\end{array}$ & of & 5 & to & 10 & 185 to $195^{\circ} \mathrm{C}$ \\
\hline $\begin{array}{l}\text { Duration } \\
\text { seconds }\end{array}$ & of & 11 & to & 60 & 140 to $150^{\circ} \mathrm{C}$ \\
\hline
\end{tabular}

These temperature rises are above the ambient of about $30^{\circ} \mathrm{C}$. Where there is an initial temperature rise involved due to loading of auxiliary windings. The current density has to be suitably reduced as premature ageing of insulation and an excessive shortening of life will occur if the limit of $140^{\circ} \mathrm{C}$ is exceeded too frequently.

\section{Rating of an Earthing Transformer}

It is customary to specify a rating for the earthing transformer. This rating for convenience is reduced to equivalent rating as it should be comparable to conventional transformer ratings. It is customary to specify the equivalent rating at required current density.

Rating of an earthing transformer $=$ (the fault rating at the time of short circuit $\times 3 /$ short circuit current density) 
For example:

If short circuit current density $=45 \mathrm{~A} / \mathrm{mm}^{2}$, then

Rating of the earthing transformer $=0.067$ times short circuit rating.

The current balancing parts like switches, busbars and terminations for a grounding transformer have to be chosen based on the fault current to be handled and the duration of

the fault currents. This is done by seeing the earthing transformer as a transformer where the current causes the same heating effect in 3 seconds.

For example:

Let the fault current per phase $=500 \mathrm{~A}$.

Let the short circuit current duration $=30$ seconds.

Let ' $\mathrm{I}_{\mathrm{e}}$ ' be the equivalent transformer current producing the same heating in 3 seconds,

then,

$$
\mathrm{I}_{\mathrm{e}}{ }^{2} \times 3=500^{2} \times 30=1580 \text { Amps }
$$

So, the current which switch, busbars and insulators must be rated for is $1580 \mathrm{Amps}$ minimum.

\section{Tappings on a Zig Zag wound Transformer}

Sometimes, customer may specify a Zig Zag wound transformer with tappings for a small variation of Zig Zag voltage. In such case tappings must be provided for, both in Zig and Zag windings and turns in Zig at a given tapping should at all times be the same as the turns in Zag at the same tapping. It must also be ensured that such tap changes are carried out by a single switch or two switches in tandem. Care must be taken to see that both Zig and Zag tappings are changed in an identical manner, as otherwise a phase shift of the resulting voltage will occur compared to normal expected voltage. This is a very important point which gives rise to interesting possibilities. This will mean that there is no ampere turns balance in the two halves of the windings in one leg, because the same current is flowing through both of them.

The calculation and derivation of impedance for a two-winding transformer with one Zig Zag winding cannot be (calculated) done in the conventional way. Since it is a polyphase system, with each leg having windings from two different phases, the system has to be resolved into its component parts (real and imaginary) from which the leakage reactance can be then calculated [6]. Under normal conditions, the magnetic flux and the voltage are in quadrature in a given phase. At, voltage maximum, the flux is zero. If the core has no residual magnetism, then switching on at the period when voltage is passing through the maximum gives no problems and the situation is exactly same as normal operation conditions [7]. The residual flux density depends upon the exact moment on the flux waveform, at which the transformer is switched off. This is also influenced by the way the core is stacked and also by the type of core steel used [8]. The compared design parameters are shown in Table 2.

Table 2. The comparison design parameters

\begin{tabular}{|l|l|l|l|}
\hline & $\begin{array}{l}\text { Delta } \\
\text { winding }\end{array}$ & $\begin{array}{l}\text { Star } \\
\text { winding }\end{array}$ & $\begin{array}{l}\text { Zig Zag } \\
\text { winding }\end{array}$ \\
\hline $\begin{array}{l}\text { Phase } \\
\text { current }\end{array}$ & $\mathrm{I} / \sqrt{ } 3$ & $\mathrm{I}$ & $\mathrm{I}$ \\
\hline Turns & $\mathrm{W}$ & $\mathrm{W} / \sqrt{3}$ & $\mathrm{~W} / 3$ \\
\hline $\begin{array}{l}\text { Area of } \\
\text { copper }\end{array}$ & $\mathrm{Ar} / \sqrt{3}$ & $\mathrm{Ar}$ & $\mathrm{Ar}$ \\
\hline
\end{tabular}

DOUBLE ZIG ZAG CONNECTIONS

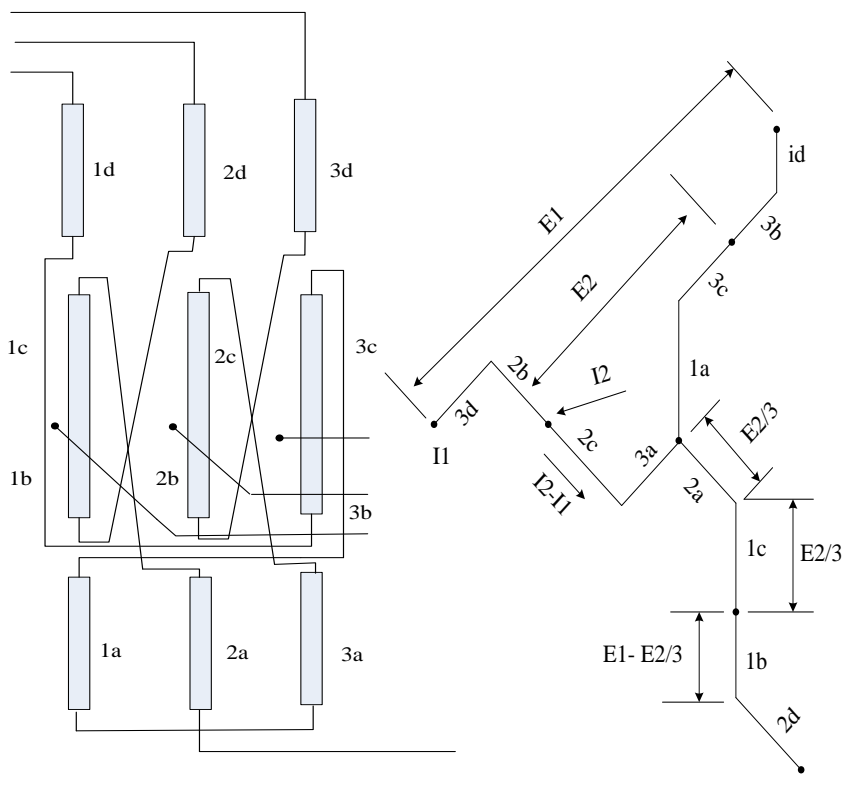

Figure 4. Double Zig Zag connection

When in addition to a 4 wire 3 phase service, a voltage transformation is also desired, the double Zig Zag connection may be used as shown in Fig.4. Auto transformers in this service unlike single $\mathrm{Zig} \mathrm{Zag}$ connection normally carry a balanced 3 phase load in addition to any unbalanced currents flowing through the 
neutral. The calculation of normal load currents in the series and shunt coils is identical with that of straight star connection.

When the voltage transformation is a small percentage, say less than $25 \%$, sometimes a single extended Zig Zag connection may be used with core type three phase units with fairly good regulation.

\section{TESTING IN FACTORY AND AT SITE}

Most of the single-phase tests that are performed on convention star or delta windings cannot be used for logical results in case of a Zig Zag winding. For example, in a star-delta transformer, the ratio of winding turns may straightaway be assured by using a single-phase voltage being applied to the particular leg in question. The same cannot be done in case of a star Zig Zag or delta Zig Zag transformers, ratio tests have to be performed by applying a three-phase voltage. For preliminary ratio verification, the Zig and Zag windings are separately compared with the other winding on the same leg, with application of single-phase voltage. [1]

The essential difference is that such single-phase voltage measurements always give the ratio of turns. Whereas ratio measurement with a polyphase voltage in case of Zig Zag always gives a voltage ratio [5]

We can also see that while performing magnetic balance tests, there is significant difference whenever one of the windings is Zig Zag. Whenever we measure the three phase magnetizing currents by exciting a star connected winding, we find that the excitation currents of the two outer legs are almost equal and individually are greater than that of the middle phase, in a core type mitred construction. But when we do the same thing on a Zig Zag winding, for a given type of connection, the magnetizing currents in and of the outer phases and in the middle phase are equal and individually are lower in value compared to the exciting current of the outer phase. This is a very interesting field for analysis.

\section{CONCLUSION}

We can thus infer that the Zig Zag connection is used to derive a neutral for a 3 phase 4 wire operation, where the neutral of the system is otherwise not available. With balanced three phase loads, the Zig Zag auto transformer carries no current except the magnetizing current. But with unbalanced loads, the unbalanced current in the neutral wire divides equally in the three phases of the auto transformer, one third flowing in each leg. The use of Zig Zag winding finds the greatest use in earthing transformers where no voltage transformation is involved. Zig Zag connected grounding transformer is much smaller than a star delta unit of the same ratings, the $\mathrm{kVA}$ parts being approximately $58 \%$ of the star delta unit. Star delta transformers of 2 to 5 seconds short circuit rating will provide satisfactory service as earthing transformers in many cases. The only advantage of star delta transformers is that it can be used as a power transformer should the need arise. Another important area of application for the Zig Zag winding is in rectifier transformers.

\section{ACKNOWLEDGEMENT}

The authors wish to thank Prof Ir Dr. Mohd Shahril Osman, Dean, School of Engineering \& Technology, UCTS, Sarawak for his support and encouragement.

\section{REFERENCES}

[1] Geno Peter, A review about testing of Distribution Transformers, International journal of Advancements in Technology, Vol 2, No 1, p.165 - 177

[2] IEC 60076-1, Power Transformers-Part 1, General 2000

[3] Geno Peter and Anli Sherine (2017) 'Induced over voltage test on transformers using enhanced Z-source inverter based circuit', Journal of Electrical Engineering, Vol. 68, No. 1,pp.378-383.

[4] ABB, Testing of Power Transformers,Zurich , 2003

[5] Geno Peter, Calculations for short circuit withstand capability of a distribution transformer, International Journal of Engineering

[6] IEC 60076-3 Power Transformers-part3: Insulation Levels, dielectric tests and external clearances in air.

[7] IEEE Guide for Induced Voltage test and Partial Discharge Measurement in Liquid-Filled Power Transformers and Shunt Reactors. IEEE C57.1131991

[8] A. Winter, P. Coors, U. Stephen, "A Mobile Transformer Test System Based on a Static Frequency Converter," in the Proceedings of HighVolt Colloquium '07, pp. 137-143, May 14-15, 2007

[9] A. Naderian Jahromi "A Fast Method for Calculation of Transformers Leakage Reactance using Energy Technique" IJE Transactions B: Applications Vol. 16, No. 1, April 2003 